Bangladesh J Med Microbiol 2019; 13 (2): 1-2

Bangladesh Society of Medical Microbiologists

\title{
Editorial
}

\section{COVID 19; Unpredicted Destiny}

\section{Professor Dr Shyamal Kumar Paul}

\author{
Professor of Microbiology, Principal, Netrokona Medical College
}

On December 2019, a cluster of cases of unexplained viral pneumonia was identified in Wuhan, China. Initially linked with the seafood market in Wuhan. The causative agent was identified as a new coronavirus, putatively named $2019-\mathrm{nCoV}$ by the WHO on January 12, 2020. On January 30, 2020, the WHO declared the outbreak of novel coronavirus a public health emergency of international concern. The International Committee on Taxonomy of Viruses renamed it as severe acute respiratory syndrome coronavirus 2 (SARS-CoV-2), and the related pneumonia as coronavirus disease 2019 (COVID-19) on February 12, 2020. ${ }^{1}$

Coronaviruses did not attract worldwide attention until the 2003 SARS epidemic, followed by the 2012 MERS outbreak and, most recently, the novel coronavirus pandemic. SARS-CoV-2 is a positive-sense, single-stranded enveloped RNA virus and is closely related to the SARS-CoV (79.6\% sequence identity) $13,[14]$. SARS-CoV-2 is closely related to bat coronavirus TG13 and is $96 \%$ identical at the whole-genome level. This suggests that SARS-CoV-2 may also have originated in bats, via an intermediate host Pangolins (99\% sequence homology). ${ }^{2}$

After an incubation period of 1-14 days COVID-19 disease may manifest either as an asymptomatic infection or a mild to severe pneumonia. At present, SARS-CoV-2-infected patients are the main source of infection. Because of the mild clinical symptoms during the incubation period, leading to the spread of infection. Asymptomatic carriers can also be a source of infection. Nosocomial transmission is also a severe problem. As of June 4, 2020 more than Half a Million health workers had been infected. ${ }^{3}$

Transmissibility of SARS-CoV-2 remains crucial for predictingthe course of the epidemic depending on the

Correspondence:

Professor Dr Shyamal Kumar Paul

Professor of Microbiology

Principal, Netrokona Medical College
$\mathrm{R}$ value. It may be very difficult to contain or control the spread of this virus without adequate preventionand control measures. The WHO declared the coronavirus outbreak to be a pandemic on March 11, 2020. Up to September 16, 2020, at least 29, 791, 038 cases of COVID 19 had been confirmed and a total of 940, 375 known deaths worldwide involving 213 countries and territories. In Bangladesh total Morbidity and Mortality up to September 16, 2020 was 342, 671 and 4823 respectively. ${ }^{4}$

To date, the gold-standard method for the laboratory diagnosis of COVID-19 is by real-time RT-PCR in nasal and throat swab samples mostly. This diagnostic method has several shortcomings. Rapid testfor Antigen and Antibodies has great potential benefit for the fast screening of SARS-CoV-2 infections to preventvirus transmission and assure the timely treatment of patients.

At present, there is no specific antiviral treatment for COVID-19, and no vaccine is availablein the markettill now. Each country should take a series of timely and effective measures to contain the spread of the pandemic and ultimately subside. Antibody testingare critical for seroprevalence studies, identify appropriate donors for convalescent sera, understanding the longevity of the antibody response after infection and post vaccination.

Though vaccine may be effective against SARS COV 2 infection, it may not be used in the first wave of the pandemic due to longer time needed to prepare. More than 100 vaccine candidates of different types are currently in preclinical development around the world, and 15 vaccines are already being tested in clinical trials. These vaccines are mostly focused on eliciting immunity against the spike glycoprotein. Concerns regarding the longevity of immune responses and for adverse effects following SARS-CoV-2 infection in immunized volunteers. Immunization may need to induce stronger immunity than natural infection. Low neutralizing antibody titres were recently reported in $30 \%$ of patients who recovered from mild infection with SARS-CoV-2.5 
Therapeutics development should focus on repurposing existing drugs, or using convalescent plasma that can rapidly be used to treat infection.

First confirmed cases of COVID-19 reinfection, recently documented in Hong Kong, as well as in the Netherlands and Belgium, have caused us to wonder how the pandemic will ever come to an end if there is no way to become immune to the novel coronavirus. So, COVID passports are not happening. And neither will herd immunity, both for natural and vaccine induced. While these new studies on reinfection open the door to more questions, the main takeaway is very clear: people who have had COVID-19 should continue to remain vigilant. Proper masking, physical distancing and appropriate cleaning of surfaces are more important than ever, because we can't necessarily fall back on herd immunity to rip off all these precautions.
1. Gorbalenya AE, et al. Severe acute respiratory syndrome-related coronavirus: The species and its viruses-a statement of the Coronavirus Study Group 2020; p. 2020.02.07.937862.

2. Zhou P, et al. A pneumonia outbreak associated with a new coronavirus of probable bat origin. Nature 2020; p. 10.1038/s41586-020-2012-7.

3. World Socialist Web Site wsws.org Published by the International Committee of the Fourth International (ICFI)

4. www.worldometers.info. Coronavirus

5. Bernheim A, et al. Chest CT Findings in Coronavirus Disease-19 (COVID-19): Relationship to Duration of Infection. Radiology 2020; p. 200463. 